\title{
Ridge Augmentation Using a Connective Tissue Graft- A Case Report
}

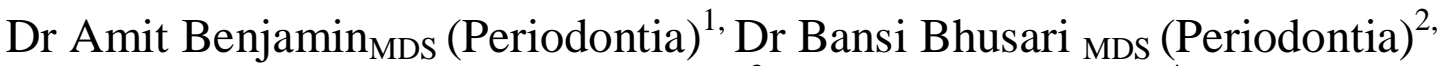 \\ Dr Jayant R Ambulgekar, ${ }^{3,}$ Dr Manan M Doshi ${ }^{4}$ \\ ${ }^{1}$ Reader, ${ }^{2}$ Head of Department, ${ }^{3}$ Post-graduate Student, ${ }^{4}$ Post-graduate Student. Department of Periodontics, \\ Yerala Medical Trust \& Research Centre's Dental College and Hospital. PG Institution. Kharghar, Navi \\ Mumbai- 410210 Maharashtra, India.
}

\begin{abstract}
A partially edentulous ridge may retain the general shape of the alveolar process. Such a ridge is traditionally referred to as a normal ridge. The smooth contours of this normal ridge create problems for restorative dentist. In a fixed bridge, the pontics frequently give the impression that they rest on the top of the ridge rather than emerge from within the alveolar process \& lack marginal gingivae \& interdental papillae. Black triangles, which almost always interfere with dentofacial esthetics, are present in the embrasure area between the pontics \& between the abutments \& the pontics. In other words, it may be difficult or impossible to produce a fixed prosthesis which truly restores the esthetics \& function of the natural dentition. The localized alveolar ridge defect refers to the volumetric deficit of the limited extend of the bone \& soft tissue within the alveolar process. Such type of ridge defect can be corrected by surgical ridge augmentation that can be accomplished by the addition of either soft or hard tissues. This article describes a procedure of surgical connective tissue augmentation of a localized deficient alveolar ridge in the maxilla, followed by fixed partial denture, enhancing the esthetics, function \& health.
\end{abstract}

Keyword: Black triangles, connective tissue augmentation, localized alveolar ridge defect, Partially edentulous ridge.

\section{Introduction}

Prosthetic rehabilitation of an isolated edentulous space in the pre-maxilla is critical, considering the high esthetic demand. [1] A deformed ridge may result from tooth extractions, advanced periodontal disease, abscess formations, etc. The deformity that exists in the ridge is directly related to the volume of root structure $\&$ associated bone that is missing or has been destroyed.

According to Siebert (1983) [2] ridge defects can be divided into three classes:

Class 1: loss of bucco-lingual width but normal apico-coronal height

Class 2: loss of apico-coronal height but normal bucco-lingual width

Class 3: a combination of loss of both height \& width of the ridge

Ridge augmentation procedures should be preceded by careful surgical-prosthetic treatment planing with joint consultations involving the surgeon \& the restorative dentist in order to attain an optimal esthetic result. The following factors should be determined prior to the initiation of the therapy:

1- Volume of tissue required to eliminate the ridge deformity

2- Type of graft procedure to be used

3- Timing of various treatment procedures

4- Design of the provisional restoration

5- Potential problems with tissue discolorations \& matching tissue color

There are high incidences of residual ridge deformity following anterior tooth loss: a majority of these are class 3 defects. [3] This clinical report describes, the soft tissue ridge augmentation to correct alveolar ridge defect combined with fixed prosthodontics to achieve maximum esthetics \& health.

\section{Case Report}

A female patient aged 48 years reported to the department of Prosthodontics, YMT Dental College, Kharghar, with the chief complaint of missing upper front teeth. (Fig.1) The dental history revealed that her left central incisor was extracted following road traffic accident 4 months back. Careful intra-oral \& radiographic examination missing maxillary left central incisor \& Seibert's class 1 defect in the anterior edentulous region. All

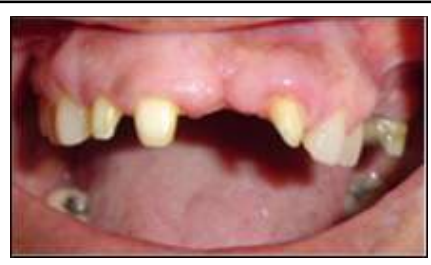

Fig 1: Pre-operative view: Facial view 
restorative options were discussed \& explained to the patient. After thorough discussion, it was decided to increase the buccolingual width of the alveolar ridge $\&$ to a certain extent ridge height. An Autogenous connective tissue graft harvested from patient's own palate was planned to be used to correct the ridge contour.

Surgical procedure: After local anesthesia was achieved, a subepithelial connective tissue graft was harvested from form the palatal side of second premolar upto mesial aspect of first

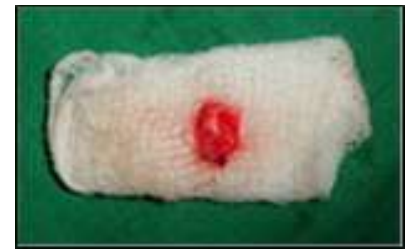

Fig 2: Harvested connective tissue from molar. (Fig.2) A horizontal internal bevel incision was made using a no. 15 surgical blade on the palate apical to the free gingival margin of the posterior teeth. 2 vertical releasing incisions were made at both ends of horizontal incision to allow for the reflection of a split-thickness flap. The length of the horizontal incision depends upon the amount of graft material to be harvested which in turn is dependent on dimensions of the concavity to be filled. The split thickness flap is reflected away from the underlying connective tissue base. The connective tissue is dissected away from the underlying bone $\&$ is used as the donor material. The split thickness flap is then replaced over bone, completely covering the bone \&sutured in place. (Fig.3) Periodontal dressing is placed on donor site \& secured with the help of a pre-adjusted surgical stent. The connective tissue is stored in a moist, sterile gauze pad for further use.

Recipient site: An attempt was made to retain as much of the lamina propria of the recipient site as possible. A no. 15 surgical blade was used to remove the epithelium. The scalpel was moved with short, saw-like strokes across the recipient site at a level approximately $1 \mathrm{~mm}$ below the outer surface of the epithelium. The least amount of connective tissue was excised. The margins of the recipient site were prepared with a butt joint. The connective tissue was placed over the alveolar ridge defect $\&$ then sutured to immobilize it in the desired position. A provisional acrylic resin bridge was fabricated \& placed over the abutment teeth. The postoperative swelling will cause the tissue to confirmto the shape of pontic. This enables the clinician to shape the soft tissue into a

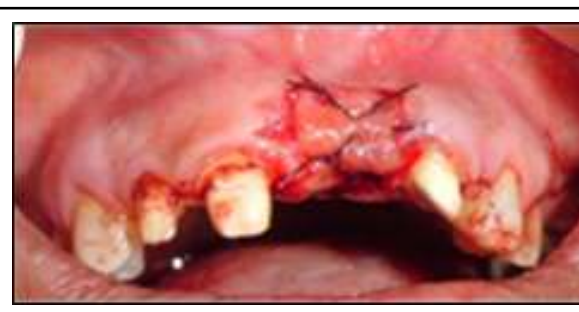

Fig 3: Connective tissue secured on recipient site form that is intended for the augmented site.

Post-surgical instructions: Systemic antibiotics \& analgesics were prescribed for 5 days post-surgically. The patient was also prescribed $0.2 \%$ chlorhexidine gluconate mouth rinse. Patient was recalled after 1 week, periodontal dressing \& sutures were removed \& the surgical area was irrigated with saline. The patient was recalled after 1 month for re-evaluation. At 1 month, there was substantial improvement in the labial contour of the alveolar ridge. On recall appointment after 2 months, the ridge form was evaluated \& considered acceptable for the permanent restoration. Four unit metal ceramic restoration was fabricated using right \& left lateral incisors to replace the maxillary left central incisor. The esthetic, function \& comfort of the restoration were adequately restored. On subsequent appointments, it was noticed that, there was no relapse of the augmented area. (Fig.4, Fig.5)
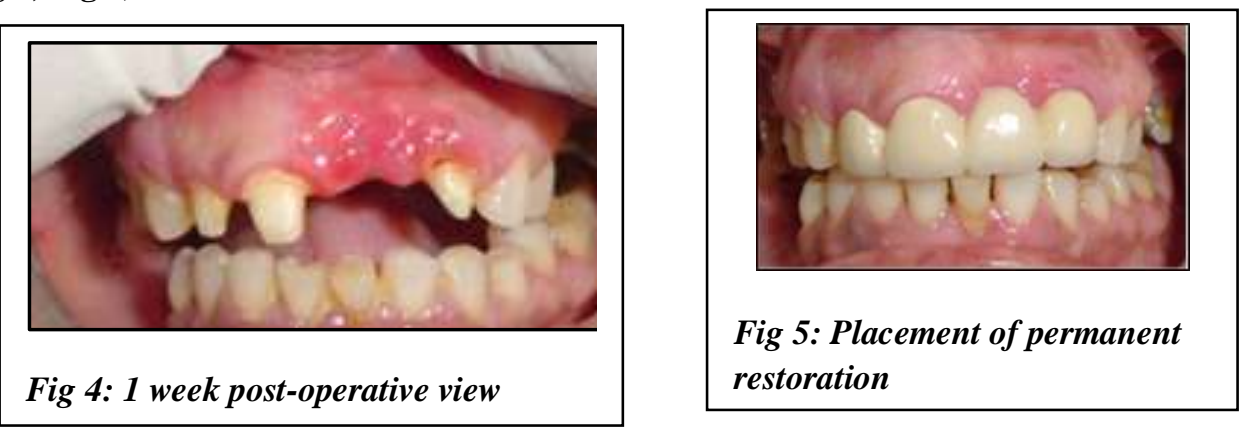

\section{Discussion}

Prosthetic treatment of localized alveolar ridge defect with a fixed prosthesis is associated with aesthetic problems i.e. "unesthetic black interdental triangles" \& technical problems i.e. "difficult to design aesthetic pontic" [4]. In the presented case, the loss of ridge width was more evident with a little loss of ridge height; hence, an autogenous connective tissue graft was used to augment the ridge the defect. A sub-epithelial 
connective tissue graft has a better chance of survival than free grafts over poor or non-vascularized areas such as a bone graft or a non-resorbable membrane [5].

\section{Conclusion}

This clinical report suggests that soft tissue augmentation with autogenous connective tissue graft harvested from the palate, in conjunction with a metal ceramic restoration, is a viable treatment to provide aesthetically acceptable contours to the alveolar ridge $\&$ an effective restoration.

\section{References}

[1] Lewis S. Anterior single tooth implant restorations. IntJPeriodontics Restorative Dent 1995; 15(1):30-41.

[2] Seibert JS. Reconstruction of deformed, partially edentulous ridges, using full thickness onlay grafts. Part II. Prosthetic /periodontal interrelationships. CompendContinEduc Dent 1983;4(6):549-562.

[3] Abrams H, Kopczyk RA, Kaplan AL. Incidence of anterior ridge deformities in partially edentulous patients. J Prosthet Dent $1987 ; 57: 191$

[4] Seibert JS, Cohen WD. Periodontal considerations in preparation for fixed and removable prosthodontics. Dent Clin North America 1987; 31: 529-555

[5] Khoury F, Happe A. The palatal subepithelial connective tissue flap method for soft tissue management to cover maxillary defects: a clinical report. Int J Oral MaxillofacImplants 2000;15:415-8 